\title{
Advantages and limitations of amino acid PET for tracking therapy response in glioma patients
}

\author{
Karl-Josef Langen ${ }^{1,2,3,6}$, Alexander Heinzel ${ }^{2,3,6}$, Philipp Lohmann ${ }^{1}$, Felix M. Mottaghy²,3,6, \\ Norbert Galldiks $1,5,6$ \\ ${ }^{1}$ Institute of Neuroscience and Medicine (INM-3, INM-4), Forschungszentrum Juelich, \\ Juelich, Germany \\ ${ }^{2}$ Dept. of Nuclear Medicine, University of Aachen, Aachen, Germany \\ ${ }^{3}$ Juelich-Aachen Research Alliance (JARA) - Section JARA-Brain \\ ${ }^{4}$ Dept. of Radiology and Nuclear Medicine, Maastricht University Medical Center \\ (MUMC+), Maastricht, The Netherlands \\ ${ }^{5}$ Dept. of Neurology ${ }^{1}$, Faculty of Medicine and University Hospital of Cologne, \\ University of Cologne, Germany \\ ${ }^{6}$ Centre of Integrated Oncology (CIO), Universities of Aachen, Bonn and Cologne, and \\ Düsseldorf, Germany
}

Running title: Monitoring of gliomas with amino acid PET

Conflicts of interest: The authors proclaim no conflicts of interest

\section{Corresponding author:}

Karl-Josef Langen, M.D.

Institute of Neuroscience and Medicine,

Forschungszentrum Juelich and

Department of Nuclear Medicine, RWTH University Clinic

52425 Juelich

Germany

Phone: +49-2461-61-5900

FAX: +49-2461-61-8261

Email: k.j.langen@fz-juelich.de 


\section{ABSTRACT}

Introduction: Today, magnetic resonance imaging (MRI) is the standard method for monitoring patients with brain tumours. The ability of conventional MRI in differentiating neoplastic tissue from non-specific, treatment-related changes after surgery, radio-, chemo- or immunotherapy, however, remains limited. Therefore, advanced MRI sequences and positron emission tomography (PET) are increasingly being considered to improve decision making.

Areas covered: PET using radiolabeled amino acids has evolved into an important diagnostic tool to overcome some of the shortcomings of conventional MRI. In view of the rapidly developing novel treatment strategies, a reliable statement on the response to therapy is becoming increasingly important. This article gives an overview of the current results of PET with radiolabelled amino acids in therapy monitoring of standard therapy as well as various innovative approaches in the treatment of patients with cerebral gliomas.

Expert commentary: Amino acid PET has proven to be helpful in therapy monitoring of gliomas, the costs are low in relation to the costs of therapy and the clinical benefit, and a widespread clinical use is highly desirable.

Key Words: cerebral glioma; PET; radiolabeled amino acids; treatment monitoring 


\section{Introduction}

Glial neoplasms of the central nervous system are the most common type of primary brain tumours and arise with an incidence of 5 to 6 new cases per 100.000 persons per year [1]. The treatment of patients with cerebral glioma consists of surgical resection and depending on the molecular profile local radiotherapy followed by concomitant and adjuvant chemotherapy with alkylating agents. Despite all efforts, the results of treatment, measured in terms of survival time, remain unsatisfactory over the decades to this day, especially in malignant gliomas. Magnetic resonance imaging (MRI) with its excellent soft tissue contrast and a high spatial resolution of 1 millimeter is currently the method of first choice for the diagnosis of brain tumours. Nevertheless, it remains difficult to differentiate brain tumours from signal abnormalities caused by non-neoplastic alterations in the tissue especially after surgery, radioand chemotherapy [2]. These shortcomings of MRI represent a major challenge in neurooncology, as therapy response assessment in gliomas is of paramount importance for an optimal treatment strategy. In particular, the timely identification of therapy failure allows the early termination of an ineffective therapy and the avoidance of side effects such as bone marrow depression, fatigue, nausea and vomiting. The change to a more efficient chemotherapy can be done with sufficient bone marrow reserve. This helps to improve patients' survival and quality of life, but also to reduce costs, as new systemic treatments are very expensive.

Therefore, a number of diagnostic approaches with a focus on metabolic and functional imaging methods are under investigation [3]. Positron emission tomography (PET) is a wellestablished method in nuclear medicine that is able to detect the distribution of radiolabelled molecules in the human body with high sensitivity and a spatial resolution of 3 - $5 \mathrm{~mm}$. Since numerous metabolic substrates, receptor ligands or pharmaceuticals can be labelled with positron-emitting isotopes such as carbon-11, nitrogen-13 or fluorine-18, PET offers great 
potential for the assessment of metabolic and physiological processes. However, radiolabelling of the molecules with short-lived positron emitters is a rather sophisticated procedure. In addition, the tracers must meet a number of conditions such as high in vivo stability, high specificity and a sufficient residence time in the target tissue in order to be clinically useful.

The most widely used PET tracer for tumour imaging is 2-[ $\left.{ }^{18} \mathrm{~F}\right]$ fluoro-2-deoxy-D-glucose (FDG) which accumulates in almost all tumours due to high glucose metabolism. In the case of gliomas, however, it is difficult to delineate the tumour tissue owing to the high rate of glucose metabolism in normal brain tissue. Although FDG has been applied in numerous brain tumour studies and provides relevant information for certain clinical questions such as grading of gliomas and prognostics, the procedure has only achieved a low priority in the clinical setting.

In the last decades, radiolabelled amino acids have emerged as the PET tracers of choice for the diagnosis of gliomas [4]. This is mainly due to the fact that the uptake of radiolabelled amino acids by normal brain tissue is relatively low and brain tumours can be distinguished from the surrounding normal tissue with high contrast. Equally important is the ability of these tracers to pass the intact blood-brain barrier (BBB) and to depict the tumour mass beyond contrast enhancement in MRI [3] and to differentiate tumour progression from nonspecific, treatment-related changes [5]. The Response Assessment in Neuro-Oncology (RANO) working group has recently recommended the use of amino acid PET imaging for brain tumour management in addition to conventional MRI at every stage of disease[4, 6].

This article gives an overview of the current results of PET with radiolabelled amino acids in therapy monitoring of standard therapy as well as various innovative approaches in the treatment of patients with cerebral gliomas. References for this Review were identified through searches of PubMed with the search terms "glioma”, "PET”, "radiolabeled amino acid”, “treatment monitoring”, “pseudoprogression”, “pseudoresponse”, 
“radiochemotherapy”, “check-point inhibitors”, “antiangiogenic”, “radiosurgery”, “from Jan 1, 1979 to Oct 30, 2019. Articles were also identified through searches of the authors' own files. Only papers published in English were reviewed. The final reference list was generated on the basis of originality and relevance to the broad scope of this Review.

\section{Limitation of conventional MRI for tracking therapy response in glioma patients}

Changes of contrast enhancement on MRI after injection of paramagnetic contrast agents are considered as an indicator of therapy response or tumour relapse [7, 8]. Contrast enhancement , however, reflects an increased permeability of the BBB and is not specific for neoplastic tissue and its changes do not necessarily reflect response to therapy or tumour relapse $[9,10]$. Following first-line chemoradiation of glioblastoma with temozolomide, progressive contrast enhancing lesions are frequently observed on MRI, which are not related to tumour progression, but are due to treatment-related effects. These lesions may remain stable or regress during further follow-up MRI without further treatment or any change of treatment [11]. This phenomenon of progressive, radiation- or chemoradiation-induced, enhancing MRI lesions in patients with malignant gliomas, with spontaneous improvement is called pseudoprogression [12, 13]. If no biopsy is taken, pseudoprogression is usually diagnosed retrospectively based on clinical findings and follow-up MRI. Pseudoprogression occurs typically within the first 12 weeks after radiotherapy with concomitant and adjuvant temozolomide for glioblastomas in 20 - $30 \%$ of the patients [13, 14] and has been incorporated into the criteria defined by the RANO working group [8]. Pseudoprogression has also been described during immunotherapy of brain metastasis or glioblastoma by blocking immune checkpoints such as cytotoxic T lymphocyte-associated antigene 4 (CTLA-4) using ipilimumab and programmed cell death 1 receptor (PD-1) using pembrolizumab or nivolumab [15-17]. 
Since the introduction of antiangiogenic agents such as bevacizumab the problem of pseudoresponse complicates the assessment of treatment response using Macdonald criteria alone [7]. Bevacizumab can rapidly decrease contrast enhancement after initiation of treatment [18], producing an apparently high response rate. Some of the improvement observed on contrast-enhanced MRI results from a rapid normalization of abnormally permeable blood vessels that restores, at least in part, the integrity of the BBB. Hence, the extent of reduction of contrast enhancement may not always reflect the true anti-tumour activity of the antiangiogenic agent [19]. Thus, the use of antiangiogenic drugs likely alters the image characteristics of enhancing tumours more effectively than that of non-enhancing ones [19]. The RANO group suggested in 2010 new recommendations for evaluating response considering FLAIR or T2 signal hyperintensity as a surrogate marker for nonenhancing tumour to help determine tumour progression [8] but these criteria do not provide quantitative values to estimate response to therapy. Furthermore, tissue alterations such as tumour-related edema, radiation injury, demyelination, ischemia, and infection can result in hyperintense FLAIR or T2 signal, which is difficult to distinguish from non-enhancing tumour [9].

\section{Radiolabeled amino acid for PET imaging of gliomas}

The longest-established amino acid tracer for PET is $\left[{ }^{11} \mathrm{C}\right.$-methyl] -L-methionine (MET) , which shows uptake via the System L amino acid transporter as well as an incorporation into protein and participation in other metabolic pathways [20]. Alpha- $\left[{ }^{11} \mathrm{C}\right]-$ methyl-L-tryptophan (AMT) has been proposed as an alternative to MET because of its involvement in the kynurenic pathway, which may play a role in immune response regulation in gliomas [20, 21]. These tracers, however, are restricted to a few neurooncological centres since the short halflife of carbon-11 (20 min) requires an onsite cyclotron [22]. Therefore, amino acids labelled with fluorine-18 (half -life of $109.8 \mathrm{~min})$ such as $\mathrm{O}-\left(2-\left[{ }^{18} \mathrm{~F}\right]\right.$-fluoroethyl) -L-tyrosine 
(FET) [23, 24], 3,4-dihydroxy- 6- [ $\left.{ }^{18} \mathrm{~F}\right]$-fluoro -L-phenylalanine (FDOPA) [25], L-[3- $\left.{ }^{18} \mathrm{~F}\right]-$ $\alpha$-methyl tyrosine (FMT) [26], and anti-1-amino-3- ${ }^{18} \mathrm{~F}$-fluorocyclobutane-1-carboxylic acid (FACBC, fluciclovine) [27] are increasingly gaining acceptance owing to logistical advantages compared with C-11-labelled amino acids. FDOPA is approved in some European Countries for clinical use, fluciclovine has orphan drug status for brain tumours in the USA [28] and FET is approved for clinical use in brain tumour imaging in France and Switzerland [29]. In Europe, the high clinical interest in this method has led to $>10,000$ FET PET scans being performed in some centres [30]. Uptake of these amino acids occurs predominantly via the transport system L for large neutral amino acids namely the subtypes LAT1 and LAT2 although other transport systems may also play a role [31-33]. Imaging of brain tumours with MET, FET and FDOPA is very similar [34-36]. Static scans made between 20 and 60 min after tracer injection are normally used for the interpretation of PET images [6]. For FET and FDOPA, the additional analysis of the time-activity curves of tracer uptake in the tumour can be helpful in differential diagnosis [37-40]. When using FDOPA, an increased uptake in the striatum has to be considered, as the molecule is a precursor of dopamine, which may cause problems in the delineation of gliomas affecting the striatum [41]. Since MRI is indespensible, amino acid PET represents an additional investigation and there is limited availability but it appears to be attractive to clinicians because the method is robust, image interpretation is simple and the metabolically active brain tumour tissue is visualized with a high tumour-to-background contrast.

\section{Amino acid PET in assessment of tumour resection}

Assessment of residual tumour following surgery is usually done by contrast-enhanced MRI which should be performed within $72 \mathrm{~h}$ after surgery since it becomes challenging to differentiate contrast-enhancing tumour tissue from treatment-related changes at later time points [42]. Contrast enhancement in early postoperative MRI, however, may not detect the full extent 
of the residual tumour since glioma tissue may extend beyond the area of contrast enhancement $[43,44]$.

Several studies have investigated the role of the amino acid PET in determining the extent of tumour resection in patients with cerebral gliomas. In a PET study using MET, residual tumour was successfully detected in 13 out of 19 pediatric brain tumours which was confirmed by repeated surgery or tumour progression in all cases [45]. The same authors reported on the prognostic role of total resection of MET-positive tissue in a group of 43 adult patients with high-grade glioma while a total removal of contrast enhancement in MRI did not correlate with survival [46]. For PET using FET the most extensive study to date in a group of 62 reported that FET PET detected residual tumour tissue more frequently than MRI [47]. In another study, the authors demonstrated a high correlation between elevated tracer uptake on the postoperative FET PET scans and the sites of subsequent tumour recurrence [48]. A recent study compared residual FET uptake after surgery in 31 patients with glioblastomas with intraoperative fluorescence after the application of 5-aminolevulinic acid (5-ALA)[49]. In that study, 13 patients showed residual FET accumulation beyond contrast enhancement in MRI and 5-ALA fluorescence. Moreover, residual tumour tissue in FET PET with a volume greater than $4.3 \mathrm{ml}$ was associated with a significant shorter overall survival time.

In an experimental study with rat gliomas, we observed increased FET uptake at the rim of the resection cavity within the first two weeks following glioma resection, especially in the first few days after surgery [50]. Since this uptake decreased in the second week after surgery, it was recommended that FET PET should be performed later than two weeks after resection. In summary, amino acid PET provides valuable information for assessing the success of glioma resection but the intervention can also lead to an increased tracer uptake that warrants further investigation. 


\section{Tracking radio- and chemotherapy in malignant glioma}

Maximum complete resection followed by radiotherapy with concomitant and adjuvant temozolomide is currently the standard of care for patients with glioblastoma [51]. The prognostic value of early changes of FET uptake after postoperative chemoradiation has been evaluated in a prospective study with glioblastoma [52]. In that study 25 patients with glioblastoma were investigated by MRI and FET PET after surgery, one week after completion of chemoradiation with temozolomide, and 6-8 weeks later. Early after completion of chemoradiation, a decrease of both the maximum and mean tumour-to-brain ratios were highly significant and independent statistical predictors for progression-free survival and overall survival. A decrease of the maximum tumour-to-brain ratios of more than $20 \%$ predicted favourable survival with a sensitivity of $83 \%$ and a specificity of $67 \%$. Oppositely, changes of the volume of contrast enhancement in MRI had no significant predictive value for survival. Examples of response assessment with FET PET in chemoradiation with temozolomide in a patient with glioblastoma is shown in Figure 1 and in a patient treated with lomustine in Figure 2. An overview on the cut-off values of amino acid PET to assess response to treatment in gliomas is shown in Table 1. Reliable monitoring of temozolomide and nitrosourea-based chemotherapy (combined procarbazine, lomustine and vincristine, or combined lomustine and vincristine or lomustine monotherapy) has also been demonstrated in patients with recurrent malignant glioma [53-56]. In another study, the value of FET PET for monitoring stereotactic brachytherapy using iodine-125 seeds was investigated in patients with recurrent malignant glioma [57]. In that study, FET PET differentiated late posttherapeutic effects after 6 months from local tumour progression with a high diagnostic accuracy but an early increase of FET uptake 3 months after seed implantation was not necessarily associated with tumour progression. The authors speculated that this observation might be caused by changes in blood-brain barrier permeability or reactive processes and recommended that changes of amino acid uptake in the early phase 
after brachytherapy of recurrent high-grade gliomas should be considered with caution. Further studies of treatment monitoring with radiolabeled amino acids during experimental treatment options for malignant gliomas such as convection-enhanced delivery of paclitaxel, intracavitary radioimmunotherapy, stereotaxy-guided laser-induced interstitial thermotherapy, immunotherapy with dendritic cell vaccination as well as adjuvant maintenance therapy with imatinib in combination with hydroxyurea reported that decreasing tracer uptake was associated with better outcome [58-62]. Furthermore, a recent study investigated the changes of FET uptake in patients with glioblastoma during therapy with tumour-treating fields (TTF) which deliver low-intensity, alternating electric fields as a locoregional intervention that inhibits cell division and causes neoplastic cell death. In two patients treated solely with TTF without any other concurrent neurooncological therapy, serial PET revealed a decrease of FET uptake over a follow-up of 6 months in accordance with stable disease [63]. Similar results have also been reported with AMT [64].

In summary, the vast majority of studies show that a response of malignant gliomas to different forms of therapy is associated with a decrease in amino acid accumulation, predicting a response to therapy earlier than MRI.

\section{Assessment of response to antiangiogenic therapy in malignant gliomas}

As outlined above, the assessment of treatment response of recurrent glioblastoma to antiangiogenic agents such as bevacizumab is complicated by the problem of pseudoresponse. Bevacizumab can rapidly decrease contrast enhancement after initiation of treatment [18], producing an apparently high response rate. The persistence of non-enhancing tumour tissue, however, is crucial for the further course of the disease and is difficult to identify by MRI. A number of studies have investigated whether amino acid PET is more reliable than MRI to assess treatment response to antiangiogenic therapy. Using FET PET, two studies with 
smaller groups of patients (10 and 11) investigated therapy monitoring of glioblastomas during anti-angiogenic therapy $[65,66]$. In both studies, changes of the metabolic tumour volume in FET PET proved to be more accurate than MRI in assessing response and predicting survival time, with the latter study also taking into account kinetic parameters of FET PET. Another study investigated therapy monitoring during combined therapy with bevacizumab and the nitrosourea lomustine in 21 patients with recurrent glioblastoma [67]. While early treatment response as assessed by RANO criteria was not predictive for overall survival, reductions of all FET PET parameters significantly predicted an overall survival of more than 9 months. The role of FDOPA PET to monitor anti-angiogenic therapy with Bevacizumab was investigated in a larger series of 30 patients with recurrent high-grade gliomas [68]. Similar to FET PET, the decrease in metabolic tumour volume predicted response to therapy more reliably than changes in MRI. In contrast to these results, a study with MET PET in 20 patients with recurrent glioblastomas showed that a decrease in tracer uptake 4 weeks after the start of anti-angiogenic therapy did not allow a prediction of therapy response [69]. A more recent study using MET and Hybrid PET/MRI in 11 patients, however, reported that both a decrease in tumor-to-brain ratio and metabolic tumor volume predicted response to anti-angiogenic therapy [70].

The prospective REGOMA phase 2 trial [71] showed an encouraging and significant survival benefit for glioblastoma patients at first progression treated with the oral multikinase inhibitor regorafenib, which has antiangiogenic properties. A recent pilot study of 5 patients revealed that FET PET allows an early diagnosis of both pseudoresponse and pseudoprogression already 8 weeks after initiation of regorafenib treatment [72].

In summary, amino acid PET has been shown to be helpful in assessing the success of antiangiogenic therapy of glioblastomas. However, further investigations assessing the value of amino acid PET in a larger number of patients undergoing antiangiogenic treatment are warranted. 


\section{Assessment of therapy response in non-enhancing low-grade glioma}

While treatment monitoring in patients with malignant glioma with MRI is usually based on volumetric changes of contrast enhancement, it is more difficult to assess therapy response in non-enhancing lower-grade gliomas (i.e. gliomas of the WHO grade II and III). Abnormalities in T2-weighted/ FLAIR MRI may represent a combination of infiltrating tumour cells, necrotic areas, tumour edema, and treatment-related leukoencephalopathy. FET PET has been used to assess effects of temozolomide chemotherapy according to the European Organisation for Research and Treatment (EORTC) protocol 22033-26033 (application of $75 \mathrm{mg} / \mathrm{m}^{2}$ temozolomide per day over 21 days in a 28-day cycle) in patients with progressive nonenhancing WHO grade II glioma [73]. In responding patients, a reduction of the FET-positive tumour volume could be observed substantially earlier than volume reductions on FLAIR sequences. Similar findings were reported by a subsequent multicenter PET study in a larger number of patients [74]. In two other studies, MET PET was applied to assess response or failure of iodine-125 seed brachytherapy in patients with WHO grade II glioma. Twelve months after brachytherapy, MET uptake was significantly reduced in the tumours, whereas glucose metabolism as assessed by FDG PET remained unchanged [75, 76]. The largest study to date included 61 patients with non-enhancing WHO grade II or III glioma who received chemotherapy with temozolomide or CCNU/procarbazine [77]. The patients were studied by FET PET before and 6 months after initiation of chemotherapy. Patients with a decrease in either metabolic tumour volume $\geq 25 \%$ and/or maximum tumour-to-brain ratios $\geq 10 \%$ exhibited a significantly longer progression-free survival than patients with stable or increasing FET uptake.

Another important aspect in the monitoring of WHO grade II gliomas is the detection of malignant transformation. In patients with low-grade gliomas following a watch-and-wait strategy, malignant progression has been reported in more than $50 \%$ within a median followup time of 7 years [78]. A study using FET PET in 27 patients with histologically proven low- 
grade glioma demonstrated that changes of tumour-to-brain ratios and kinetic parameters of FET uptake identified malignant progression with a significantly higher diagnostic accuracy than changes of contrast enhancement in MRI [79].

Summarizing, similar to the results in malignant gliomas, amino acid PET proved to be a reliable tool for tracking therapy response non-enhancing low-grade gliomas.

\section{Differentiation of tumour recurrence and treatment-related changes}

The differentiation between tumour relapse and therapy-induced changes represents one of the most frequent indications for the use of the amino acid PET in clinical practice. Although it cannot be regarded directly as part of therapy monitoring, it plays an important role in this context and should be considered in this review. As outlined above, pseudoprogression in MRI is a frequent problem following first-line chemoradiation of glioblastoma with temozolomide. FET PET has been shown to differentiate progressive gliomas from pseudoprogression with high accuracy $[80,81]$. Concerning the differentiation of recurrent tumour versus radionecrosis or other treatment-related changes, several studies have reported an accuracy ranging from 80 to $90 \%$ for FET or FDOPA PET [5, 82-86]. For MET PET, a recent meta-analysis including 891 patients reported on a pooled sensitivity and specificity of $88 \%$ and $85 \%$ in the differential diagnosis of glioma recurrence [87]. For AMT, a first study in a series of 22 patients reported on a $100 \%$ accuracy to differentiate between recurrent glioma and radiation injury [88]. Thus, amino acid PET has developed as a reliable method to distinguish the frequent therapy-induced structural changes in MRI from tumour progression, which is of crucial importance in therapy monitoring.

\section{Cost-effectiveness of treatment monitoring using amino acid PET}

The scientifically documented utility of amino acid PET for tracking therapy response in glioma patients emphasizes its use in clinical practice in order to avoid overtreatment and 
unnecessary side effects. However, since additional PET examinations are costly, it is important to consider cost efficiency of PET. Some studies addressed the cost-effectiveness using a model-based approach relying on the best available evidence. One study estimated the effectiveness and cost-effectiveness of the addition of FET PET to structural MR imaging for the management of antiangiogenic treatment in patients with recurrent high-grade glioma compared with MR imaging alone [89]. The use of FET PET resulted in a number needed to diagnose of 2.4, indicating that 3 additional patients have to be diagnosed to avoid one wrong diagnosis. From the perspective of the Statutory Health Insurance in Germany the incremental cost-effectiveness ratio of FET PET/MR imaging compared with MR imaging alone was at least 5,725 € per life-year. Thus, costs of FET PET appear to be well justified by their clinical utility. A recent study analyzed cost-effectiveness of follow-up FET PET performed on patients with glioblastoma after surgery and before initiation of temozolomide maintenance treatment [90]. Based on cost calculations from the perspective of the National Institute for Health and Disability Insurance (NIHDI) in Belgium two cost-effectiveness ratios were determined for overall survival and progression-free survival rates. Both of these calculations yielded very similar results: incremental cost-effectiveness ratios of $1,365.86$ and 1,357.38 $€$, respectively, for each identified non-responder. The authors concluded that FET PET is a valuable tool for predicting the treatment responses of patients with glioblastomas to followup temozolomide maintenance treatment under consideration of cost-effectiveness.

\section{Conclusions}

Treatment monitoring of gliomas is still associated with significant shortcomings in the age of high-resolution structural imaging with MRI, which can be solved in many cases by the use of amino acid PET. This method is helpful to assess the extent of tumour resection, to assess therapy response to chemoradiation and other treatment options in patients with malignant gliomas as well as in non-enhancing lower-grade gliomas. The method helps to overcome 
pseudoprogression and pseudoresponse, two frequent diagnostic problems of conventional MRI that respectively occur at an early stage after chemoradiation and antiaangiogenic treatment. The costs of amino acid PET appear to be well justified in relation to the clinical benefit. A further establishment of the method in clinical practice is desirable, in order to avoid overtreatment and unnecessary side effects.

\section{Expert opinion}

Molecular imaging of cerebral gliomas with amino acid PET is becoming more and more available for clinical use. The more widespread use of amino acid PET for the management of patients with brain tumours has been strongly recommended by the RANO group [4, 6].

In view of the rapidly developing novel treatment strategies, a reliable statement on the response to therapy is becoming increasingly important. The available literature convincingly demonstrates the potential of amino acid PET to track therapy response in cerebral gliomas but the number of studies is still too small to draw final conclusions.

Concerning the evaluation of tumour resection some studies observed advantages of amino acid PET over early postoperative MRI.

Tracking therapy response with amino acid PET is best established in malignant gliomas, especially in chemoradiation with temozolomide as well as anti-angiogenic therapy with bevacizumab. Here, the improved identification of pseudoprogression and pseudoresponse plays an important role.

Experience with experimental forms of therapy suggest that molecular imaging with amino acid PET will be helpful to track novel therapeutic approaches in neurooncology.

Furthermore, in non-enhancing lower-grade gliomas, amino acid PET proved to be a reliable tool for tracking therapy response, which is of particular importance as the efficiency of monitoring with conventional MRI is limited in these tumours. 
Overall, the data situation has to be improved and an intensification of research is necessary. Unfortunately, amino acid PET is not yet available at every oncologic center treating brain tumours. A limitation for the widespread clinical use of amino acid PET in treatment monitoring of gliomas remains the lack of approval and reimbursement by national insurances. State authorities usually require a proven patient-relevant benefit and/or demonstration of cost-efficiency. This is usually established by randomized, blinded, two-arm multicentre studies, in which patients with brain tumours are diagnosed and monitored with MRI only or amino acid PET and MRI. Such studies take many years but there is little interest for commercial support, because the synthesis of most amino acid tracers has been published and no patent is pending. Furthermore, such studies rise ethical questions since $50 \%$ of the patients would not be given access to a diagnostic method that provides valuable additional information according to current literature. Fortunately, there are exceptions in some countries for university clinics and specialized centers to use amino acid PET in patients with cerebral gliomas for clinical purposes. Owing to the relatively low incidence of cerebral gliomas, this provides access to amino acid PET for a large part of the population in some countries in Western Europe.

Functional or molecular MRI methods, such as perfusion-weighted and diffusion-weighted imaging and proton magnetic resonance spectroscopy are currently under clinical evaluation to overcome the shortcomings of conventional MRI. The fact that amino acid PET is widely used in centres that have full access to the spectrum of functional and molecular MRI methods emphasizes the value of amino acid PET beyond these alternative MRI methods. Nevertheless, reliable treatment monitoring can probably best be achieved by a combination of different imaging techniques [91]. The advent of hybrid PET/MRI offers the opportunity to investigate several parameters in a ‘one-stop shop'. Using hybrid PET/MRI scanners, several studies have been started to investigate these aspects and should lead to meaningful results in the near future. In addition, novel radiopharmaceuticals for PET are being tested, which could 
lead to further progress in these issues. Although hybrid PET/MRI has practical advantages and is more convenient for patients, the higher cost of these systems must be weighed against the additional effort of sequential tests.

Thus, it is to be expected that more effective treatment monitoring will become available in the next years leading to a considerable benefit for brain tumour patients. 


\section{Article Highlights}

- Amino acid PET provides significant additional information for tracking therapy response in glioma patients

- Amino acid PET requires additional scanning but is robust and attractive for clinicians in neurooncology because of easy scan reading

- Amino acid PET in therapy monitoring of gliomas is cost efficient and well justified to avoid overtreatment and unnecessary side effects.

- Improvement of therapy monitoring in gliomas is necessary because therapy is extremely expensive and efficient use of these therapies is mandatory 


\section{FIGURES}

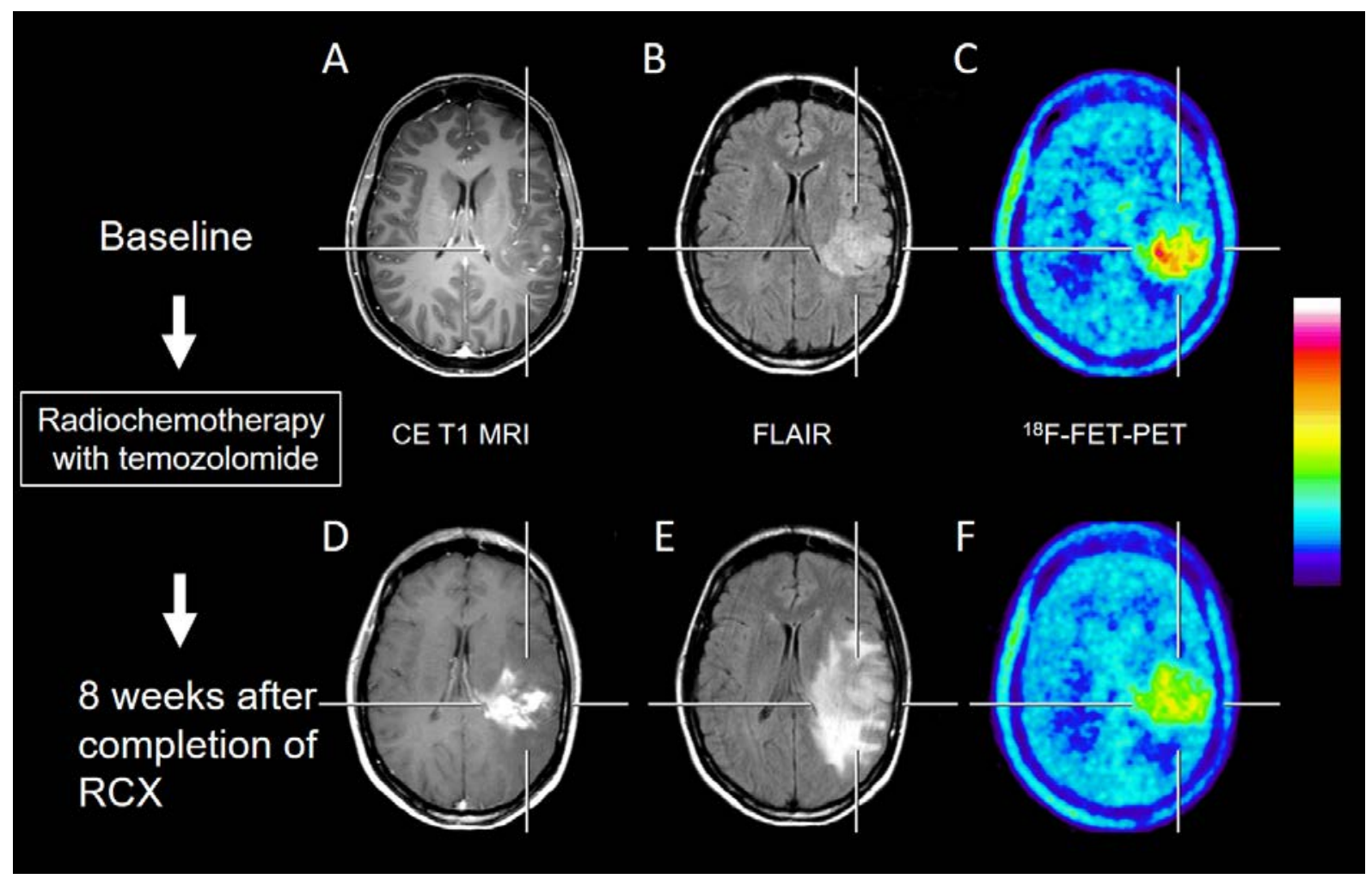

Figure 1: Therapy monitoring in a 47-year-old patient with glioblastoma. Contrast-enhanced MRI (CE T1 MRI), fluid-attenuated inversion recovery (FLAIR) MRI and FET PET (A-C) at baseline and 8 weeks after completion of chemoradiation with concomitant and adjuvant temozolomide (RCX)(D-F). Enlargement of contrast enhancement and FLAIR abnormalities suggest tumour progression whereas FET PET shows decreased metabolic activity compared to initial FET PET demonstrating pseudoprogression which was confirmed by follow-up MRI at 3 months after RCX. 


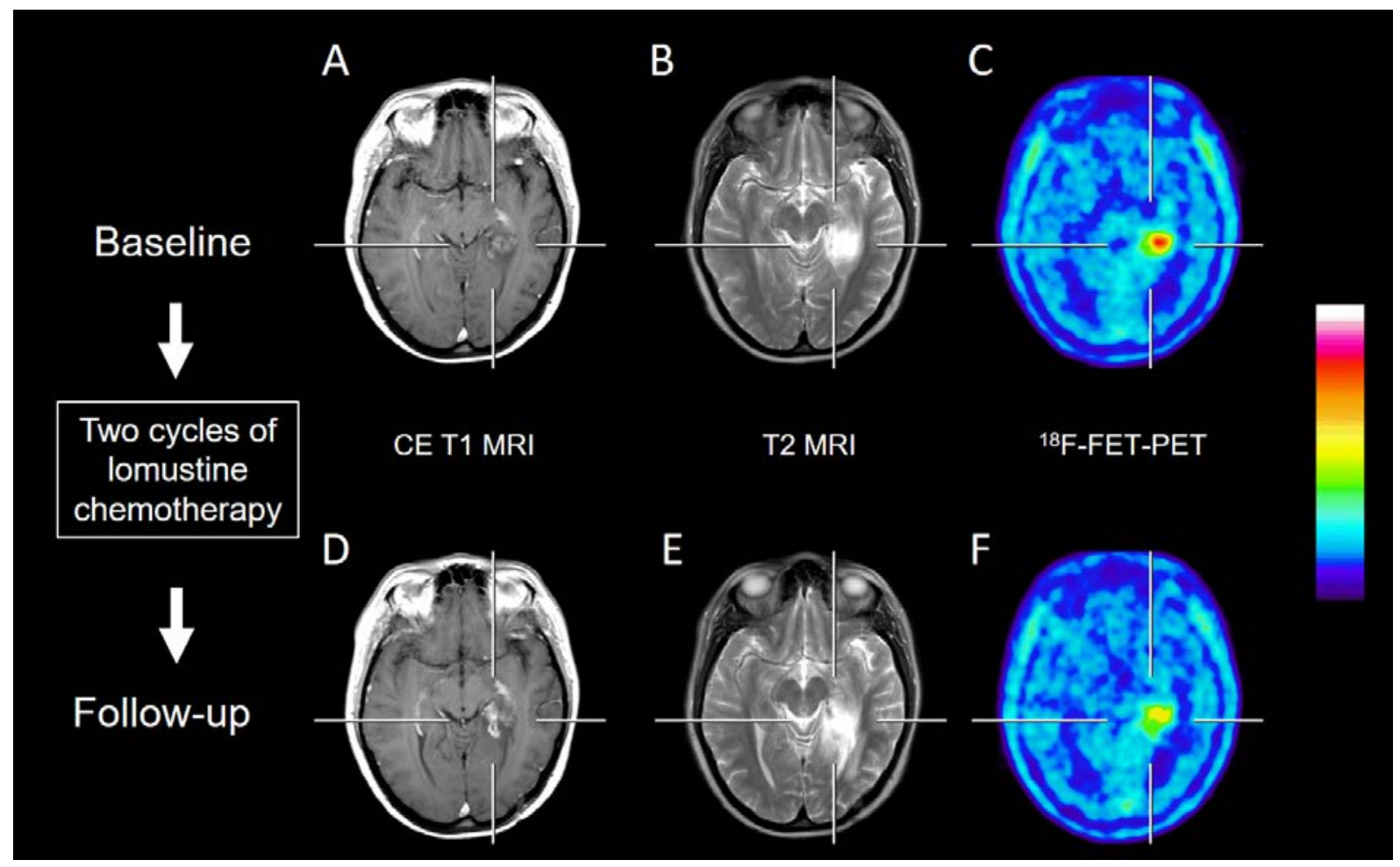

Figure 2: Therapy monitoring in a 31-year-old patient with a recurrent anaplastic astrocytoma in the left parahippocampal region after brachytherapy and radiotherapy with concomitant and adjuvant temozolomide. Contrast-enhanced MRI (CE T1 MRI), T2-weighted MRI and FET PET (A-C) at baseline and 4 months later after 2 cycles of lomustine chemotherapy (D-F). Enlargement of contrast enhancement and the T2 signal suggest tumour progression whereas FET PET indicates a reduction of metabolic activity and response to therapy. 
Table 1: Overview of cut-off values of amino acid PET to assess response to treatment

\begin{tabular}{|c|c|c|c|c|}
\hline Therapy /diagnostic problem & Tracer & Parameter & Cut off & Reference \\
\hline \multirow{2}{*}{$\begin{array}{l}\text { Differentiation of tumour recurrence/progress versus } \\
\text { treatment-related changes }\end{array}$} & FET & $\begin{array}{l}\text { TBRmax }(\mathrm{ROI} \varnothing 1.6 \mathrm{~cm}) \text {; } \\
\text { TTP }\end{array}$ & $>1.9-2.3 ;$ TTP $<45$ min & {$[5,80,81]$} \\
\hline & MET & $\mathrm{TBR}_{\max }$ & $>1.9$ & {$[92,93]$} \\
\hline Malignant transformation of low-grade gliomas & FET & $\mathrm{TBR}_{\max }$ & increase $>33 \%$ & [79] \\
\hline Radiochemotherapy with temozolomide in glioblastoma & FET & $\mathrm{TBR}_{\max }$ & decrease $>20 \%$ & [52] \\
\hline $\begin{array}{l}\text { Antiangiogenic therapy with Bevacizumab//rinotecan in } \\
\text { glioblastoma }\end{array}$ & FET & BTV & decrease $>45 \%$ & {$[65,66]$} \\
\hline $\begin{array}{l}\text { Temozolomide or lomustine/procarbazine in non-enhancing } \\
\text { gliomas }\end{array}$ & FET & $\mathrm{TBR}_{\max }$ BTV & decrease $>10 \% ;>25 \%$ & [77] \\
\hline Temozolomide in malignant gliomas & MET & $\mathrm{TBR}_{\max }(\mathrm{ROI} \varnothing 1.2 \mathrm{~cm}$ & decrease $>5 \%$ & [53] \\
\hline Antiangiogenic therapy with bevacizumab in recurrent gliomas & MET & $\mathrm{TBR}_{\text {mean }}$ & decrease $>25 \%$ & [70] \\
\hline
\end{tabular}

Abbreviations: $\mathrm{TBR}_{\max } / \mathrm{TBR}_{\text {mean }}=$ Maximum/mean tumour to brain ratio: $\mathrm{TTP}=$ Time-to-peak in time activity curve; $\mathrm{MTV} / \mathrm{BTV}=$ metabolic or biological tumour volume; ROI = Region-of-interest 


\section{REFERENCES}

Papers of special note have been highlighted as either of interest $(\bullet)$ or of considerable interest $(\bullet)$ to readers.

1. Weller M, van den Bent M, Tonn JC, Stupp R, Preusser M, Cohen-Jonathan-Moyal E, et al. European Association for Neuro-Oncology (EANO) guideline on the diagnosis and treatment of adult astrocytic and oligodendroglial gliomas. The lancet oncology. 2017;18:e315-e29.

2. Galldiks N, Langen KJ. Applications of PET imaging of neurological tumors with radiolabeled amino acids. Q J Nucl Med Mol Imaging. 2015;59:70-82.

3. Langen KJ, Galldiks N, Hattingen E, Shah NJ. Advances in neuro-oncology imaging. Nature reviews Neurology. 2017;13(5):279-289.

** Comparative overview of the contribution of PET and advanced MR methods in brain tumor diagnostics

4. Albert NL, Weller M, Suchorska B, Galldiks N, Soffietti R, Kim MM, et al. Response Assessment in Neuro-Oncology working group and European Association for NeuroOncology recommendations for the clinical use of PET imaging in gliomas. Neuro-oncology. 2016;18:1199-208.

\section{** Recommendation of the RANO group concerning the use of PET in brain tumour} diagnostics

5. Galldiks N, Stoffels G, Filss C, Rapp M, Blau T, Tscherpel C, et al. The use of dynamic O-(218F-fluoroethyl)-l-tyrosine PET in the diagnosis of patients with progressive and recurrent glioma. Neuro-oncology. 2015;17:1293-300.

6. Law I, Albert NL, Arbizu J, Boellaard R, Drzezga A, Galldiks N, et al. Joint EANM/EANO/RANO practice guidelines/SNMMI procedure standards for imaging of gliomas using PET with radiolabelled amino acids and [(18)F]FDG: version 1.0. European journal of nuclear medicine and molecular imaging. 2019;46:540-57.

\section{* Procedure guidelines for PET imaging of brain tumours}

7. Macdonald DR, Cascino TL, Schold SC, Jr., Cairncross JG. Response criteria for phase II studies of supratentorial malignant glioma. J Clin Oncol. 1990;8:1277-80.

8. Wen PY, Macdonald DR, Reardon DA, Cloughesy TF, Sorensen AG, Galanis E, et al. Updated response assessment criteria for high-grade gliomas: response assessment in neurooncology working group. J Clin Oncol. 2010;28:1963-72.

9. Ahluwalia MS, Wen PY. Antiangiogenic therapy for patients with glioblastoma: current challenges in imaging and future directions. Expert Rev Anticancer Ther. 2011;11:653-6.

10. Dhermain FG, Hau P, Lanfermann H, Jacobs AH, van den Bent MJ. Advanced MRI and PET imaging for assessment of treatment response in patients with gliomas. Lancet neurology. 2010;9:906-20.

11. Taal W, Brandsma D, de Bruin HG, Bromberg JE, Swaak-Kragten AT, Smitt PA, et al. Incidence of early pseudo-progression in a cohort of malignant glioma patients treated with chemoirradiation with temozolomide. Cancer. 2008;113:405-10. 
12. Brandes AA, Franceschi E, Tosoni A, Blatt V, Pession A, Tallini G, et al. MGMT promoter methylation status can predict the incidence and outcome of pseudoprogression after concomitant radiochemotherapy in newly diagnosed glioblastoma patients. J Clin Oncol. 2008;26:2192-7.

13. Brandsma D, van den Bent MJ. Pseudoprogression and pseudoresponse in the treatment of gliomas. Current opinion in neurology. 2009;22:633-8.

14. Brandsma D, Stalpers L, Taal W, Sminia P, van den Bent MJ. Clinical features, mechanisms, and management of pseudoprogression in malignant gliomas. The lancet oncology. 2008;9:453-61.

15. Okada H, Weller M, Huang R, Finocchiaro G, Gilbert MR, Wick W, et al. Immunotherapy response assessment in neuro-oncology: a report of the RANO working group. The lancet oncology. 2015;16:e534-e42.

16. Preusser M, Lim M, Hafler DA, Reardon DA, Sampson JH. Prospects of immune checkpoint modulators in the treatment of glioblastoma. Nature reviews Neurology. 2015;11:504-14.

17. Wolchok JD, Hoos A, O'Day S, Weber JS, Hamid O, Lebbe C, et al. Guidelines for the evaluation of immune therapy activity in solid tumors: immune-related response criteria. Clinical Cancer Research. 2009;15:7412-20.

18. Vredenburgh JJ, Desjardins A, Herndon JE, 2nd, Marcello J, Reardon DA, Quinn JA, et al. Bevacizumab plus irinotecan in recurrent glioblastoma multiforme. J Clin Oncol. 2007;25:4722-9.

19. Ahluwalia MS, Wen PY. Antiangiogenic therapy for patients with glioblastoma: current challenges in imaging and future directions. Expert Rev Anticancer Ther. 2011;11:653-6.

20. Herholz K. Brain Tumors: An Update on Clinical PET Research in Gliomas. Seminars in nuclear medicine. 2017;47:5-17.

21. Chugani DC, Muzik O, Chakraborty P, Mangner T, Chugani HT. Human brain serotonin synthesis capacity measured in vivo with alpha-[C-11]methyl-L-tryptophan. Synapse. 1998;28:33-43.

22. Langen KJ, Galldiks N. Update on amino acid PET of brain tumours. Current opinion in neurology. 2018;31:354-61.

23. Wester HJ, Herz M, Weber W, Heiss P, Senekowitsch-Schmidtke R, Schwaiger M, et al. Synthesis and radiopharmacology of O-(2-[18F]fluoroethyl)-L-tyrosine for tumor imaging. Journal of Nuclear Medicine. 1999;40:205-12.

24. Hamacher K, Coenen HH. Efficient routine production of the 18F-labelled amino acid O-218F fluoroethyl-L-tyrosine. Applied radiation and isotopes : including data, instrumentation and methods for use in agriculture, industry and medicine. 2002;57:853-6.

25. Heiss WD, Wienhard K, Wagner R, Lanfermann H, Thiel A, Herholz K, et al. F-Dopa as an amino acid tracer to detect brain tumors. Journal of Nuclear Medicine. 1996;37:1180-2. 
26. Inoue T, Shibasaki T, Oriuchi N, Aoyagi K, Tomiyoshi K, Amano S, et al. 18F alpha-methyl tyrosine PET studies in patients with brain tumors. Journal of Nuclear Medicine. 1999;40:399-405.

27. Shoup TM, Olson J, Hoffman JM, Votaw J, Eshima D, Eshima L, et al. Synthesis and evaluation of [18F]1-amino-3-fluorocyclobutane-1-carboxylic acid to image brain tumors. Journal of of Nuclear Medicine. 1999;40:331-8.

28. Approvals SODDa. Fluciclovine. US Food \& Drug Administration website. 2015.

29. Swissmedic. Swiss Agency for Therapeutic Products. Journal Swissmedic. 2014;13:651.

30. Langen KJ, Tonn JC, Weller M, Galldiks N. Letter to the Editor: "The role of imaging in the management of progressive glioblastoma. A systematic review and evidence-based clinical practice guideline" [ $\mathrm{J}$ Neurooncol 2014; 118:435-460]. Journal of neuro-oncology. 2014;120:665-6.

31. Okubo S, Zhen HN, Kawai N, Nishiyama Y, Haba R, Tamiya T. Correlation of L-methyl11C-methionine (MET) uptake with L-type amino acid transporter 1 in human gliomas. Journal of neuro-oncology. 2010;99:217-25.

32. Youland RS, Kitange GJ, Peterson TE, Pafundi DH, Ramiscal JA, Pokorny JL, et al. The role of LAT1 in (18)F-DOPA uptake in malignant gliomas. Journal of neuro-oncology. 2013;111:11-8.

33. Habermeier A, Graf J, Sandhofer BF, Boissel JP, Roesch F, Closs EI. System L amino acid transporter LAT1 accumulates O-(2-fluoroethyl)-L-tyrosine (FET). Amino acids. 2015;47:335-44.

34. Becherer A, Karanikas G, Szabo M, Zettinig G, Asenbaum S, Marosi C, et al. Brain tumour imaging with PET: a comparison between [18F]fluorodopa and [11C]methionine. European journal of nuclear medicine and molecular imaging. 2003;30:1561-7.

35. Grosu AL, Astner ST, Riedel E, Nieder C, Wiedenmann N, Heinemann F, et al. An Interindividual Comparison of O-(2- [(18)F]Fluoroethyl)-L-Tyrosine (FET)- and L-[Methyl(11)C]Methionine (MET)-PET in Patients With Brain Gliomas and Metastases. International journal of radiation oncology, biology, physics. 2011;81:1049-58.

36. Kratochwil C, Combs SE, Leotta K, Afshar-Oromieh A, Rieken S, Debus J, et al. Intraindividual comparison of (18)F-FET and (18)F-DOPA in PET imaging of recurrent brain tumors. Neuro-oncology. 2014;16:434-40.

37. Calcagni ML, Galli G, Giordano A, Taralli S, Anile C, Niesen A, et al. Dynamic O-(2[18F]fluoroethyl)-L-tyrosine (F-18 FET) PET for glioma grading: assessment of individual probability of malignancy. Clinical nuclear medicine. 2011;36:841-7.

38. Popperl G, Kreth FW, Herms J, Koch W, Mehrkens JH, Gildehaus FJ, et al. Analysis of 18FFET PET for grading of recurrent gliomas: is evaluation of uptake kinetics superior to standard methods? Journal of nuclear medicine. 2006;47:393-403.

39. Weckesser M, Langen KJ, Rickert CH, Kloska S, Straeter R, Hamacher K, et al. O-(2[18F]fluorethyl)-L-tyrosine PET in the clinical evaluation of primary brain tumours. European journal of nuclear medicine and molecular imaging. 2005;32:422-9. 
40. Ginet M, Zaragori T, Marie PY, Roch V, Gauchotte G, Rech F, et al. Integration of dynamic parameters in the analysis of (18)F-FDopa PET imaging improves the prediction of molecular features of gliomas. European journal of nuclear medicine and molecular imaging. 2019. Sep 16. doi: 10.1007/s00259-019-04509-y. [Epub ahead of print]

41. Cicone F, Filss CP, Minniti G, Rossi-Espagnet C, Papa A, Scaringi C, et al. Volumetric assessment of recurrent or progressive gliomas: comparison between F-DOPA PET and perfusion-weighted MRI. European journal of nuclear medicine and molecular imaging. 2015;42:905-15.

42. Forsting M, Albert FK, Kunze S, Adams HP, Zenner D, Sartor K. Extirpation of glioblastomas: MR and CT follow-up of residual tumor and regrowth patterns. AJNR American journal of neuroradiology. 1993;14:77-87.

43. Scott JN, Brasher PM, Sevick RJ, Rewcastle NB, Forsyth PA. How often are nonenhancing supratentorial gliomas malignant? A population study. Neurology. 2002;59:947-9.

44. Lohmann P, Stavrinou P, Lipke K, Bauer EK, Ceccon G, Werner JM, et al. FET PET reveals considerable spatial differences in tumour burden compared to conventional MRI in newly diagnosed glioblastoma. European journal of nuclear medicine and molecular imaging. 2019;46:591-602.

45. Pirotte B, Levivier M, Morelli D, Van Bogaert P, Detemmerman D, David P, et al. Positron emission tomography for the early postsurgical evaluation of pediatric brain tumors. Child's nervous system. 2005;21:294-300.

46. Pirotte BJ, Levivier M, Goldman S, Massager N, Wikler D, Dewitte O, et al. Positron emission tomography-guided volumetric resection of supratentorial high-grade gliomas: a survival analysis in 66 consecutive patients. Neurosurgery. 2009;64:471-81.

\section{** Study on the role of MET PET in the assessment of tumor resection}

47. Buchmann N, Klasner B, Gempt J, Bauer JS, Pyka T, Delbridge C, et al. (18)F-Fluoroethyl-lThyrosine Positron Emission Tomography to Delineate Tumor Residuals After Glioblastoma Resection: A Comparison with Standard Postoperative Magnetic Resonance Imaging. World neurosurgery. 2016;89:420-6.

48. Buchmann N, Gempt J, Ryang YM, Pyka T, Kirschke JS, Meyer B, et al. Can Early Postoperative O-(2-(18F)Fluoroethyl)-l-Tyrosine Positron Emission Tomography After Resection of Glioblastoma Predict the Location of Later Tumor Recurrence? World neurosurgery. 2019;121:e467-e74.

49. Muther M, Koch R, Weckesser M, Sporns P, Schwindt W, Stummer W. 5-Aminolevulinic Acid Fluorescence-Guided Resection of 18F-FET-PET Positive Tumor Beyond Gadolinium Enhancing Tumor Improves Survival in Glioblastoma. Neurosurgery. 2019;85:E1020-E9.

50. Geisler S, Stegmayr C, Niemitz N, Lohmann P, Rapp M, Stoffels G, et al. Treatment-Related Uptake of O-(2-(18)F-Fluoroethyl)-l-Tyrosine and l-[Methyl-(3)H]-Methionine After Tumor Resection in Rat Glioma Models. Journal of of Nuclear Medicine. 2019;60:1373-9.

51. Stupp R, Mason WP, van den Bent MJ, Weller M, Fisher B, Taphoorn MJ, et al. Radiotherapy plus concomitant and adjuvant temozolomide for glioblastoma. N Engl J Med. 2005;352:987-96. 
52. Galldiks N, Langen K, Holy R, Pinkawa M, Stoffels G, Nolte K, et al. Assessment of treatment response in patients with glioblastoma using [18F]Fluoroethyl-L-Tyrosine PET in comparison to MRI. Journal of Nuclear Medicine. 2012;53:1048-57.

\section{** Prospective study on the use of FET PET for treatment monitoring of glioblastoma with standard radiochemotherapy}

53. Galldiks N, Kracht LW, Burghaus L, Thomas A, Jacobs AH, Heiss WD, et al. Use of 11Cmethionine PET to monitor the effects of temozolomide chemotherapy in malignant gliomas. European journal of nuclear medicine and molecular imaging. 2006;33:516-24.

54. Galldiks N, Kracht LW, Burghaus L, Ullrich RT, Backes H, Brunn A, et al. Patient-tailored, imaging-guided, long-term temozolomide chemotherapy in patients with glioblastoma. Molecular imaging. 2010;9:40-6.

55. Herholz K, Kracht LW, Heiss WD. Monitoring the effect of chemotherapy in a mixed glioma by C-11-methionine PET. Journal of Neuroimaging. 2003;13:269-71.

56. Galldiks N, Langen KJ. Amino Acid PET - An Imaging Option to Identify Treatment Response, Posttherapeutic Effects, and Tumor Recurrence? Frontiers in neurology. 2016;7:120.

57. Jansen NL, Suchorska B, Schwarz SB, Eigenbrod S, Lutz J, Graute V, et al. [18F]fluoroethyltyrosine-positron emission tomography-based therapy monitoring after stereotactic iodine-125 brachytherapy in patients with recurrent high-grade glioma. Molecular imaging. 2013;12:137-47.

58. Pöpperl G, Goldbrunner R, Gildehaus FJ, Kreth FW, Tanner P, Holtmannspotter M, et al. O(2-[18F]fluoroethyl)-L-tyrosine PET for monitoring the effects of convection-enhanced delivery of paclitaxel in patients with recurrent glioblastoma. Eur J Nucl Med Mol Imaging. 2005;32:1018-25.

59. Pöpperl G, Götz C, Rachinger W, Schnell O, Gildehaus FJ, Tonn JC, et al. Serial O-(2[(18)F]fluoroethyl)-L:-tyrosine PET for monitoring the effects of intracavitary radioimmunotherapy in patients with malignant glioma. European journal of nuclear medicine and molecular imaging. 2006;33:792-800.

60. Galldiks N, Ullrich R, Schroeter M, Fink GR, Kracht LW. Imaging biological activity of a glioblastoma treated with an individual patient-tailored, experimental therapy regimen. $\mathrm{J}$ Neurooncol. 2009;93:425-30.

61. Galldiks N, von Tempelhoff W, Kahraman D, Kracht LW, Vollmar S, Fink GR, et al. 11CMethionine positron emission tomographic imaging of biologic activity of a recurrent glioblastoma treated with stereotaxy-guided laser-induced interstitial thermotherapy. Molecular imaging. 2012;11:265-71.

62. Kristin Schmitz A, Sorg RV, Stoffels G, Grauer OM, Galldiks N, Steiger HJ, et al. Diagnostic impact of additional O-(2-[18F]fluoroethyl)-L-tyrosine ((18)F-FET) PET following immunotherapy with dendritic cell vaccination in glioblastoma patients. British journal of neurosurgery. 2019:1-7.

63. Ceccon G, Lazaridis L, Stoffels G, Rapp M, Weber M, Blau T, et al. Use of FET PET in glioblastoma patients undergoing neurooncological treatment including tumour-treating 
fields: initial experience. European journal of nuclear medicine and molecular imaging. 2018;45:1626-35.

64. Bosnyak E, Barger GR, Michelhaugh SK, Robinette NL, Amit-Yousif A, Mittal S, et al. Amino Acid PET Imaging of the Early Metabolic Response During Tumor-Treating Fields (TTFields) Therapy in Recurrent Glioblastoma. Clinical nuclear medicine. 2018;43:176-9.

65. Hutterer M, Nowosielski M, Putzer D, Waitz D, Tinkhauser G, Kostron H, et al. O-(2-18Ffluoroethyl)-L-tyrosine PET predicts failure of antiangiogenic treatment in patients with recurrent high-grade glioma. Journal of Nuclear Medicine. 2011;52:856-64.

\section{* First study using FET PET in antiangiogenic therapy}

66. Galldiks N, Rapp M, Stoffels G, Fink GR, Shah NJ, Coenen HH, et al. Response assessment of bevacizumab in patients with recurrent malignant glioma using [18F]Fluoroethyl-Ltyrosine PET in comparison to MRI. European journal of nuclear medicine and molecular imaging. 2013;40:22-33.

67. Galldiks N, Dunkl V, Ceccon G, Tscherpel C, Stoffels G, Law I, et al. Early treatment response evaluation using FET PET compared to MRI in glioblastoma patients at first progression treated with bevacizumab plus lomustine. European journal of nuclear medicine and molecular imaging. 2018;45:2377-86.

68. Schwarzenberg J, Czernin J, Cloughesy TF, Ellingson BM, Pope WB, Grogan T, et al. Treatment Response Evaluation Using 18F-FDOPA PET in Patients with Recurrent Malignant Glioma on Bevacizumab Therapy. Clinical Cancer Research. 2014;20:3550-9.

\section{* Largest study using FDOPA PET in treatment monitoring of antiangiogenic therapy}

69. Beppu T, Terasaki K, Sasaki T, Sato Y, Tomabechi M, Kato K, et al. MRI and 11C-methyl-Lmethionine PET Differentiate Bevacizumab True Responders After Initiating Therapy for Recurrent Glioblastoma. Clinical nuclear medicine. 2016;41:852-7.

70. Deuschl C, Moenninghoff C, Goericke S, Kirchner J, Koppen S, Binse I, et al. Response assessment of bevacizumab therapy in GBM with integrated 11C-MET-PET/MRI: a feasibility study. European journal of nuclear medicine and molecular imaging. 2017;44:1285-95.

71. Lombardi G, De Salvo GL, Brandes AA, Eoli M, Ruda R, Faedi M, et al. Regorafenib compared with lomustine in patients with relapsed glioblastoma (REGOMA): a multicentre, open-label, randomised, controlled, phase 2 trial. The lancet oncology. 2019;20:110-9.

72. Galldiks N, Werner J-M, Tscherpel C, Fink GR, Langen K-J. Imaging findings following regorafenib in malignant gliomas: FET PET adds valuable information to anatomical MRI. Neuro-Oncology Advances. 2019. October 20. doi:10.1093/noajnl/vdz038. Epub ahead of print.

73. Wyss M, Hofer S, Bruehlmeier M, Hefti M, Uhlmann C, Bartschi E, et al. Early metabolic responses in temozolomide treated low-grade glioma patients. J Neurooncol. 2009;95:87-93.

74. Roelcke U, Wyss MT, Nowosielski M, Ruda R, Roth P, Hofer S, et al. Amino acid positron emission tomography to monitor chemotherapy response and predict seizure control and progression-free survival in WHO grade II gliomas. Neuro-oncology. 2016;18:744-51. 


\section{** Largest study using MET PET in treatment monitoring of low-grade gliomas}

75. Voges J, Herholz K, Holzer T, Würker M, Bauer B, Pietrzyk U, et al. 11C-methionine and 18F-2-fluorodeoxyglucose positron emission tomography: a tool for diagnosis of cerebral glioma and monitoring after brachytherapy with 125I seeds. Stereotact Funct Neurosurg. 1997;69:129-35.

76. Würker M, Herholz K, Voges J, Pietrzyk U, Treuer H, Bauer B, et al. Glucose consumption and methionine uptake in low-grade gliomas after iodine-125 brachytherapy. Eur J Nucl Med. 1996;23:583-6.

77. Suchorska B, Unterrainer M, Biczok A, Sosnova M, Forbrig R, Bartenstein P, et al. (18)FFET-PET as a biomarker for therapy response in non-contrast enhancing glioma following chemotherapy. Journal of neuro-oncology. 2018;139:721-30.

\section{** Largest study using FET PET in treatment monitoring of low-grade gliomas}

78. Jakola AS, Myrmel KS, Kloster R, Torp SH, Lindal S, Unsgard G, et al. Comparison of a strategy favoring early surgical resection vs a strategy favoring watchful waiting in low-grade gliomas. JAMA. 2012;308:1881-8.

79. Galldiks N, Stoffels G, Ruge MI, Rapp M, Sabel M, Reifenberger G, et al. Role of O-(2-18Ffluoroethyl)-L-tyrosine PET as a diagnostic tool for detection of malignant progression in patients with low-grade glioma. Journal of Nuclear Medicine. 2013;54:2046-54.

80. Kebir S, Fimmers R, Galldiks N, Schafer N, Mack F, Schaub C, et al. Late Pseudoprogression in Glioblastoma: Diagnostic Value of Dynamic O-(2-[18F]fluoroethyl)-L-Tyrosine PET. Clinical Cancer Research. 2016;22:2190-6.

81. Galldiks N, Dunkl V, Stoffels G, Hutterer M, Rapp M, Sabel M, et al. Diagnosis of pseudoprogression in patients with glioblastoma using O-(2-[18F]fluoroethyl)-L-tyrosine PET. European journal of nuclear medicine and molecular imaging. 2015;42:685-95.

82. Karunanithi S, Sharma P, Kumar A, Khangembam BC, Bandopadhyaya GP, Kumar R, et al. 18F-FDOPA PET/CT for detection of recurrence in patients with glioma: prospective comparison with 18F-FDG PET/CT. European journal of nuclear medicine and molecular imaging. 2013;40:1025-35.

83. Rachinger W, Goetz C, Popperl G, Gildehaus FJ, Kreth FW, Holtmannspotter M, et al. Positron emission tomography with O-(2-[18F]fluoroethyl)-l-tyrosine versus magnetic resonance imaging in the diagnosis of recurrent gliomas. Neurosurgery. 2005;57:505-11;

84. Maurer GD, Brucker DP, Stoffels G, Filipski K, Filss CP, Mottaghy FM, et al. (18)F-FET PET imaging in differentiating glioma progression from treatment-related changes - a singlecenter experience. Journal of Nuclear Medicine. 2019. Sep 13 doi:10.2967/jnumed.119.234757. [Epub ahead of print]

85. Bashir A, Mathilde Jacobsen S, Molby Henriksen O, Broholm H, Urup T, Grunnet K, et al. Recurrent glioblastoma versus late posttreatment changes: diagnostic accuracy of O-(2[18F]fluoroethyl)-L-tyrosine positron emission tomography (18F-FET PET). Neurooncology. 2019. Sep 6. doi:10.1093/neuonc/noz166. [Epub ahead of print] 
86. Youland RS, Pafundi DH, Brinkmann DH, Lowe VJ, Morris JM, Kemp BJ, et al. Prospective trial evaluating the sensitivity and specificity of 3,4-dihydroxy-6-[18F]-fluoro-Lphenylalanine (18F-DOPA) PET and MRI in patients with recurrent gliomas. Journal of neuro-oncology. 2018;137:583-91.

87. Xu W, Gao L, Shao A, Zheng J, Zhang J. The performance of 11C-Methionine PET in the differential diagnosis of glioma recurrence. Oncotarget. 2017;8:91030-9.

88. Alkonyi B, Barger GR, Mittal S, Muzik O, Chugani DC, Bahl G, et al. Accurate differentiation of recurrent gliomas from radiation injury by kinetic analysis of alpha-11Cmethyl-L-tryptophan PET. Journal of Nuclear Medicine. 2012;53:1058-64.

89. Heinzel A, Muller D, Langen KJ, Blaum M, Verburg FA, Mottaghy FM, et al. The use of O(2-18F-fluoroethyl)-L-tyrosine PET for treatment management of bevacizumab and irinotecan in patients with recurrent high-grade glioma: a cost-effectiveness analysis. Journal of Nuclear Medicine. 2013;54:1217-22.

90. Baguet T, Verhoeven J, De Vos F, Goethals I. Cost-Effectiveness of [(18)F] Fluoroethyl-LTyrosine for Temozolomide Therapy Assessment in Patients With Glioblastoma. Front Oncol. 2019;9:814.

91. Lohmann P, Werner JM, Shah NJ, Fink GR, Langen KJ, Galldiks N. Combined Amino Acid Positron Emission Tomography and Advanced Magnetic Resonance Imaging in Glioma Patients. Cancers. 2019;11: E153

92. Tripathi M, Sharma R, Varshney R, Jaimini A, Jain J, Souza MM, et al. Comparison of F-18 FDG and C-11 methionine PET/CT for the evaluation of recurrent primary brain tumors. Clinical nuclear medicine. 2012;37:158-63.

93. Deuschl C, Kirchner J, Poeppel TD, Schaarschmidt B, Kebir S, El Hindy N, et al. (11)C-MET PET/MRI for detection of recurrent glioma. European journal of nuclear medicine and molecular imaging. 2018;45:593-601. 